\title{
Enhanced Classroom Presenter
}

\author{
K. Jelemenská, P. Koine and P. Čičák \\ Institute of Computer Systems and Networks, Faculty of Informatics and Information Technologies \\ Slovak University of Technology Bratislava \\ Ilkovičova 3 \\ 84216 Bratislava, Slovakia \\ jelemenska@fiit.stuba.sk; xkoine@stuba.sk; cicak@fiit.stuba.sk
}

\begin{abstract}
Nowadays perhaps the most widespread teaching style at the university lectures is based on slides presentation using computer and data projector. Compared to the previously used "blackboard and chalk" style, data projectors brought a substantial loss of interactivity between students and teacher into the teaching process. Students are often taking notes without even thinking what they are writing down. This can in no way develop their creative thinking. Recently the "active learning" teaching style emerged and is becoming more and more preferable. To use this style in conjunction with computer and data projector the interactive presentation system is one of the basic assumptions. Among the available open-source presentation systems supporting interaction the Classroom Presenter, developed at the University of Washington, seems to be one of the most promising. The article describes the extensions that were designed and implemented to this presentation tool at the Faculty of Informatics and Information Technologies, Slovak University of Technology in Bratislava mainly to enhance the editing, import, and export possibilities of the tool.
\end{abstract}

\section{INTRODUCTION}

Human interacts with his environment on a daily basis. Even as children, we learn through communication with other people and through processing information others show us. It is the easiest way for us to learn. To completely understand a subject however, we need to discuss.

Over the years the teaching styles have evolved from the "blackboard and chalk" through the "whiteboard and board marker," "overhead projector and transparencies" to the nowadays most widespread data projector presentations. Although the data projector brought a lot of advantages like cleaner environment, better visibility especially in large classrooms, possibility of projection screen duplication, higher flexibility etc. a number of issues occurred as well. The most important include oneway static transfer of information from teacher towards students, uncomfortable ways of active entries into the presentation, and static or no access to presentation materials.

A lot of lecturers noticed the declining students' engagement and an inability of students to follow the lecture content. When taking the notes most of the students do it without knowing what they are writing. There was an urgent need to make the lectures more attractive. However, the loss of the possibility to annotate the presentations using handwriting made it difficult. The only way of attracting students' attention was the laser pointer which is hardly visible in huge lecture halls. These were the main reasons that encouraged the development of interactive presentation systems that would support things like attention catching marks for emphasizing slide content, writing illustrating examples, annotating diagrams, drawings or pictures as well as promoting the audience interaction [1]. That means the system that can give audience the possibility to interfere directly with the presentation itself. Therefore, as the whole product it can help students to understand the problem by presenting the subject, discussing it and interacting with it.

\section{RELATED WORK}

There are several interactive presentation systems that give us the functionality mentioned above. They include freeware, shareware, as well as the commercial applications [2].

\section{A. DyKnow Vision}

DyKnow [3] stands for Dynamic Knowledge. Created at the DePauw University by Dr. Berque to help students learn new things it was later commercialized and its functionality widely extended. It has client-server architecture and supports both wired and wireless networks. The whole communication is encapsulated into the HTTP protocol. DyKnow Vision allows students authentication and provide some monitoring capabilities. To get students' feedback, teacher can create little tests that are sent to the client applications where the students solve them and send them back to the teacher. Another interesting possibility is to give the student a control over the teacher's working space to fill in some information.

\section{B. Classroom Presenter}

Classroom Presenter (CP) was designed and implemented by students at the University of Washington [4]. It is an open source tool that has many useful functions. It has the object oriented architecture. The slides are divided into several sheet layers: image sheet layer, text sheet layer and ink sheet layer. Several commonly used stylus types are supported e.g. pen, highlighter, erase pen etc. CP provides quick poll (voting for an answer to multi-choice question) as well as the possibility to send the whole-slide solution to the teacher.

Concerning the possibility to extend the system there is one important disadvantage: the $\mathrm{CP}$ software 
documentation is far from perfect therefore making any changes would not be an easy task.

\section{Ubiquitous Presenter}

This system is an extension to Classroom Presenter version 2 (the latest available version is 3.1). It has been developed at the University of California, San Diego [5]. One of the main extensions was the implementation of a web based java applet which allows the user to connect to the presentation using a web browser. Students' submissions are sent to the teacher's application through a central server. It supports ink as well as other annotation techniques and a new submission style - short message service (SMS) should be available soon.

\section{NetSupport School}

NetSupport School [6] is not exactly an interactive presentation system. Its main purpose is the class management. It can manage the whole computers and their running processes. However, it has some capabilities that allow the users to present slides and to save the presentation and solutions as a Portable Document Format (PDF) journal. It also allows students to submit their contributions and to perform the tests.

The main disadvantage is the initial high cost investment into the application itself and into the class equipment with tablet PC or other computers.

\section{E. InkSurvey}

InkSurvey [7] is a completely web based application, freeware tool. The teacher can ask questions during the presentation and the students can answer them using ink or keyboard. When submitting the answers the students can choose how certain they are with the answers. However, only Mozilla Firefox web browser is fully supported.

\section{F. eBeam Projection}

eBeam Projection by Luidia [8] is more a hardware solution than a software one since to be functional a hardware package is needed. This contains an interactive stylus that behaves like a mouse, an eBeam receiver that can be mounted on almost any surface (wall, whiteboard etc.) to turn it into an interactive whiteboard, and a data projector. The receiver catches the stylus position and the actions on the projected screen and transfers it back to the computer. eBeam Projection is accompanied with an eBeam Interact software package including an eBeam Scrapbook - the presentation tool with content sharing and collaboration features using an Internet connection.

The comparison of the mentioned tools is summarized in Table I.

TABLE I

COMPARISON OF INTERACTIVE PRESENTATION SYSTEMS

\begin{tabular}{|c|c|c|c|c|c|c|}
\hline & $\begin{array}{l}\text { DyKnow } \\
\text { Vision }\end{array}$ & $\begin{array}{l}\text { Classroom } \\
\text { Presenter }\end{array}$ & $\begin{array}{l}\text { Ubiquitous } \\
\text { Presenter }\end{array}$ & $\begin{array}{l}\text { NetSupport } \\
\text { School }\end{array}$ & InkSurvey & eBeam Projection \\
\hline Hardware compatibility & All & All & All & All & All & Need of eBeam HW \\
\hline Central server & By producer & No & Yes & Yes & Yes & $\begin{array}{l}\text { Local host or by } \\
\text { producer }\end{array}$ \\
\hline Controlled authentication & Yes & No & No & Yes & Yes & By producer \\
\hline $\begin{array}{l}\text { Microsoft Power Point } \\
\text { integration }\end{array}$ & Yes & No & No & Yes & No & Partialy \\
\hline Stylus preasure support & Yes & Yes & Yes & - & - & Yes \\
\hline Network communication & Yes & Yes & Yes & Yes & Yes & Partialy \\
\hline Multimonitor support & Yes & Yes & Yes & Yes & - & No \\
\hline Price per license & $\$ 30-\$ 108$ & Free of charge & MSR-SSLA & $\begin{array}{c}\$ 546.00 \text { per } 10 \\
\text { licenses }\end{array}$ & Free of charge & Free with eBeam HW \\
\hline
\end{tabular}




\section{ClassRoOM PRESENTER EXTENSIONS}

After experiencing several interactive presentation applications we found out that none of them satisfies our expectations completely. All the tested applications can be characterized by poor import possibilities. If ever a tool supported other than its own natural format then it was PowerPoint presentation. So the lecture materials prepared in other format can not be reused with these systems. Since we believe this is an important restriction we decided to concentrate on this issue. There were two possibilities: to design and implement a completely new interactive presentation tool or to improve one of the existing ones. The University of Washington's Classroom Presenter 3.1 (CP3) was chosen for this purpose [11] simply because it is the only open source tool. Based on the CP3 license it can be extended and redistributed by anyone providing that the license conditions will be met. We realized the potential of this application consisting mainly in its quality interaction support.

\section{A. Classroom Presenter Original Features}

The main feature of Classroom Presenter 3.1 is its role based choosing system. It supports two different role types. The first one is standalone, the second one is networked. In the standalone type you can choose to work only as an instructor.

In the networked type, there are 3 roles. The first one is the student role. As the role implies it was designed to provide the students with a possibility to connect to the presentation. After the successful connection they can download the presentation, browse it, and annotate it with their own notes. They can also be allowed to send their solution slides to the teacher and to answer the quick polls.

The second role is the instructor role. This role is identical to the standalone instructor. The only difference is that it creates a TCP based server which the students are connecting to.

The last role is called public display. This role was created only to give the teacher the possibility not to be connected directly to the projector. In this case another computer with public display role is connected to the projector and is used as a middleman to the teacher who can roam freely in the classroom.

Other features of $\mathrm{CP} 3$ include pen stylus and highlighter stylus. The user can change the stylus color and in case it is supported by the computer technology the application can recognize the stylus pressure too.

As it was mentioned above, CP3 has a completely object based architecture. The object slide consists of multiple sheet layers: image sheet layer, text sheet layer, and ink sheet layer. The layers themselves are objects too.

Image sheet layer - holds an image which can be resized and moved. Resizing keeps the aspect ratio, therefore we cannot change only its height or width. This is the bottommost layer.

Text sheet layer - gives us the opportunity to insert normal text into the presentation. Font, size and color can be set.
Ink sheet layer - stores all the strokes the user draws. It is the topmost layer, therefore it is always redrawn as the last one and is always visible.

CP3 supports dual monitor output and is able to export slides in the form of images and Hypertext Markup Language (HTML) documents.

As an input it is able to open its native format and the Microsoft Power Point presentations. Primarily devoted to presenting and annotating the presentations prepared in advance in Microsoft Office PowerPoint, the presentation editing capabilities of $\mathrm{CP} 3$ are very poor compared to other environments. It does not even support the basic editing features like slide movement or copying which one would say are indispensable when reviewing the older presentations.

Among the editing options we can find the zoom out button. Zooming out scales down the slide size and an extra space is available for additional notes.

Except for PowerPoint presentations there are no other import possibilities. So the materials prepared in other formats can not be reused with Classroom Presenter.

\section{B. Enhanced Classroom Presenter}

While using the CP3 during lectures presentation several issues occurred that could or even should be solved to improve the comfort and the efficiency of the Presenter. Some extensions were designed and implemented into the CP3 in the frame of diploma thesis $[9,10]$ and will be described in this section.

\section{Role Selection Wizard}

The first one was the role selection wizard. This wizard simplifies the role choice using a step by step user interface (Fig.1). The original login window was not very practical on the monitors with low resolution. It caused the login window to show up outside the working area. This wizard corrects the problem. It allows teacher to simply create a TCP session and to start the presentation. The students can then easily join the selected session.

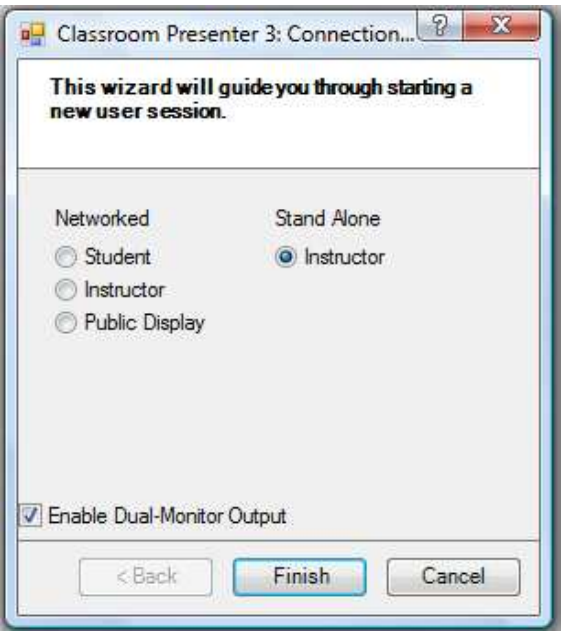

Fig.1. Role selection wizard. 


\section{Presentation Publishing}

Another feature that was implemented is the presentation publishing. To accomplish that we export the current presentation to the PDF format, fill in the server IP address and publish the document. Of course, to publish the document the central server has to be created first. The server allows the teacher to divide students into classes and to sort the published materials. The connection to the server needs an authorization which recognizes the privilege level of the logging in person. The paths to the documents are not absolute, instead they are computed using an MD5 hash algorithm.

\section{Import and Export Extensions}

The possibility to import and export the $P D F$ documents was implemented as well. To export the PDF document we took the advantage of $\mathrm{CP} 3$ to export slides as images. We take these images and create a PDF document from them. Just before the document creation, we can add some metadata

like author, title etc. The resolution of the used images can also be changed setting up the DPI (dots per inch).

Another document type we can import is the Microsoft Word document. The supported formats are .doc, .docx and .rtf. To open these types of documents we need to have Microsoft Word installed. The supported versions are Word 2003, Word 2007 and Word 2010 Beta edition.

The document can be opened using one of the three options (see Fig. 2):

1) 1:1 style: this opens the document in CP3 as a one to one copy i.e. as we can see it in Microsoft Word. This style does not allow the slide content to be edited afterwards.

2) PPT style: this style is very similar to the Power Point presentation. The Word document is resized, rescaled and then used to create the slides. The resulting slide content cannot be edited either.

3) Editable text: the last style enables to open the Word document as an editable text. It uses the PPT style format, but it is then parsed to $\mathrm{CP} 3$. For each document page we parse its text and create a text sheet layer, for each picture in the page we create an image sheet layer and for each table we create an additional text sheet layer.

\section{Basic Editing Features}

An effort has been made to implement some basic editing features. The original CP3 did not support the slide

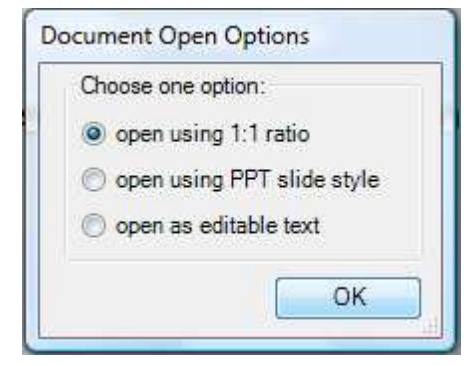

Fig.2. Word document open options. position change. Therefore, we changed the default behavior of the mouse when entering slide miniature. According to the original behavior to see the slide preview it was necessary to hold down a mouse button. We changed it to a more intuitive behavior. Entering the slide miniature the preview is shown immediately. After five seconds without any movement the preview closes automatically.

Holding down a mouse button over a slide miniature the slide movement is possible. We can move it down or up to another slide position and then release the mouse button to move the slide to the new position. To show where the slide is going to be moved a graphic marker was added showing us the current position.

The original CP3 did not support the copy/paste function either. As it is widely used, the functionality of $\mathrm{CTRL}+\mathrm{C}$ and CTRL+Vshortcuts was added as well. To differentiate between copying the whole slide or just one sheet the stylus selection is used. In case the pen or the highlighter stylus is selected the shortcut CTRL+C will add the whole slide into the clipboard. In case the text stylus is selected the copy shortcut will place the topmost text sheet layer into the clipboard. Finally, in case of image stylus selection the copy shortcut will place the topmost image sheet layer into the clipboard.

The paste functionality is made more versatile. According to the clipboard content the corresponding slide or sheet will be pasted. The possibility to paste images from the clipboard directly to the slide was added too. In this case the new image sheet layer is added into the current slide. This feature makes for example the use of snipping tool more comfortable.

The next new feature is the possibility to zoom in. This feature gives us the opportunity to show the audience some detail in the slide (see Fig.3). It can be useful when for example a large picture should be included into the presentation and the small details are not perfectly visible.

The last new feature is the reflector stylus. This stylus will create a gray half transparent layer on the top of the slide. Using a mouse or other selecting device we can then select the region we want to see normally. In other words a

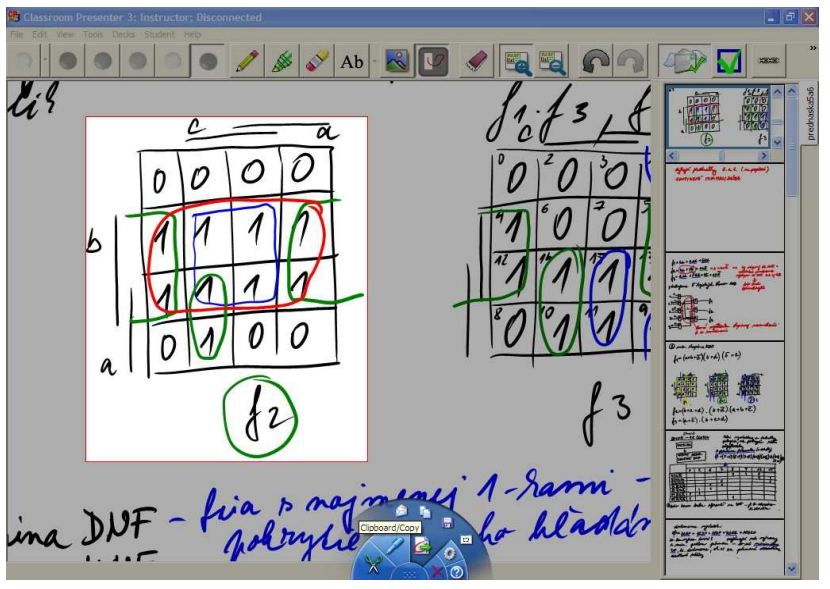

Fig.3. Snipping tool on zoomed in slide. 
window is created through which the selected part is visible. Two reflector styles were implemented: rectangle style and custom shape style.

With rectangle style stylus the selection is reshaped to a rectangle which is then removed from the layer. With custom shape reflector stylus the selection is transformed into a closed curve which is then removed from the layer. Fig. 4 shows us the custom shape reflector style. This allows the teacher to show the students only a part of the slide and to focus on a specific problem.

Two new tools were appended to the original Toolbar Reflector and Zoom in and the icon of the former Zoom out tool was modified. The differences are illustrated in Fig. 5.

\section{CONCLUSION}

In this paper the interactive presentation system called Enhanced Classroom Presenter was presented. The system is based on the Classroom Presenter 3.1 [11] using its basic functionality and expanding it to meet our needs. The main extensions include: PDF documents import and export, Word document import, presentation publishing, zoom in, reflector, and slide movement functions. The possibility to copy the whole slide or just the image or text sheet layers and to paste the content of the clipboard into the presentation or current slide is also supported. The role selection was simplified, replacing the original one with a step by step role selection wizard.

The Enhanced Classroom Presenter is an open source product just like the original one and is used for lectures presentation at the Faculty of Informatics and Information Technologies, Slovak University of Technology in Bratislava.

\section{ACKNOWLEDGMENT}

The support by Slovak Science Grant Agency (VEGA 1/0649/09 "Security and reliability in distributed computer systems and mobile computer networks") is gratefully acknowledged.

\section{REFERENCES}

[1] R. Anderson, R. Anderson, L. McDowell, B. Simon, "Use of Classroom Presenter in Engineering Courses," in FIE '05 Proc. of the 35th Annual Conference Frontiers in Education, IEEE, 2005, pp. T1A - 1-6.

[2] F. Kowalski, J. Williams, R. Reed, J. Vanides, "Transforming teaching and learning using Tablet PCs," A panel discussion using Tablet PCs, in Proc. of the 2007 American Society for Engineering Educational Annual Conference and Exposition.

[3] D. Berque, "An Evaluation of a Broad Deployment of DyKnow

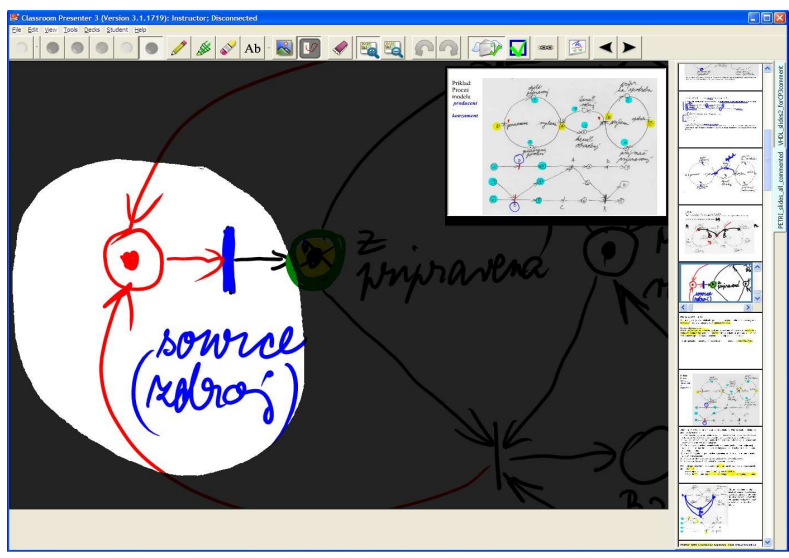

Fig.4. Custom shape reflector on zoomed in slide.

Software to Support Note Taking and Interaction Using Pen-Based Computers," in Journal of Computing Sciences in Colleges Vol. 21, No.6, June 2006, pp.204-216.

[4] R. Anderson et al. "Classroom Presenter: A Classroom Interaction System for Active and Collaborative Learning," in The Impact of Tablet PCs and Pen-based Technology on Education: vignettes, evaluations, and future directions, Berque, D.A., Prey, J.C., Reed, R.H. (Eds.), West Lafayette: Purdue University Press, 2006, 200 p. ISBN: 978-1-55753-434-7. pp. 21-30.

[5] M. Wilkerson,W.G. Griswold, B. Simon, "Ubiquitous Presenter: Increasing Student Access and Control in a Digital Lecturing Environment," in SIGCSE'05, February 23-27, 2005, St. Louis, Missouri, USA.

[6] "NetSupport School v.10.5: Complete Classroom Management,"available online at

http://www.wyse.com/solutions/education/NetSupportSchool-105-brochure.pdf

[7] F. Kowalski, S. Kowalski, E. Hoover, "Using InkSurvey: A Free Web-Based Tool for Open-Ended Questioning to Promote Active Learning and Real-Time Formative Assessment of Tablet PCEquipped Engineering Students," in Proc. of the 2007 American Society for Engineering Educational Annual Conference and Exposition, $12 \mathrm{p}$.

[8] Luidia Inc. "eBeam Projection: Transform Projections Into Interactive Workspaces," available online at http://www.luidia.com/products/ebeam-projection.html

[9] J. Hrubša, "Interactive Presentation System," [Interaktívny prezentačný systém]. Master theses, Faculty of Informatics and Information Technology, Slovak Univerzity of Technology Bratislava, 2009, 84 p.

[10] P. Koine, "Interactive Presentation System," [Interaktívny prezentačný systém] Master theses, Faculty of Informatics and Information Technology, Slovak Univerzity of Technology Bratislava, 2010, $52 \mathrm{p}$.

[11] University of Washington: Classroom Presenter. [Online, accessed March 2010]. Available at

$\mathrm{http}: / /$ classroompresenter.cs.washington.edu/
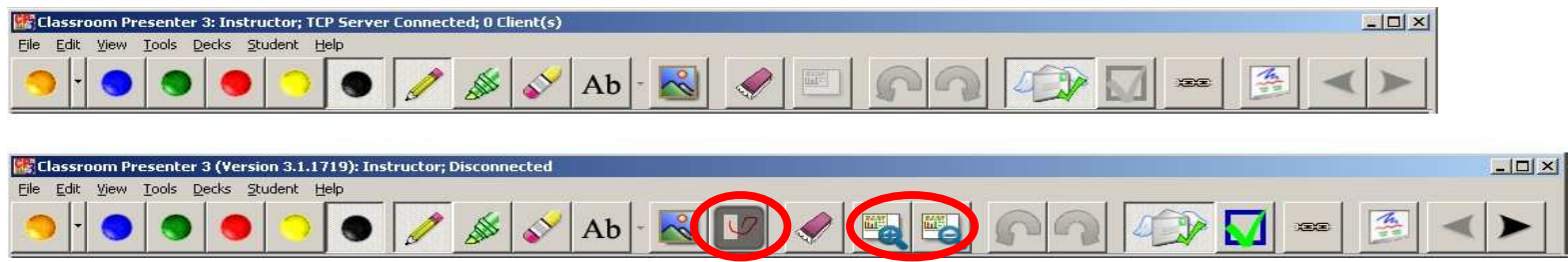

Fig. 5. Toolbar extension in Enhanced Classroom Presenter. 